\title{
An Introduction to Genomic Imprinting and Parent of Origin Effects
}

\author{
J.G. Hall, E. Lopez-Rangel \\ Department of Pediatrics, University of British Columbia and Children's Hospital, Van- \\ couver, Canada
}

\section{INTRODUCTION}

Recent developments in molecular genetics and cytogenetics have allowed for better understanding of the inheritance and expression of genes. Many newly recognized mechanisms such as genomic imprinting, mosaicism, allelic expansion, cytoplasmic inheritance and uniparental disomy have been recognized to play an important role in human heredity.

Genomic imprinting refers to differences in the phenotype which are observed depending on whether the gene was inherited from the father or from the mother. Genomic imprinting is a difficult concept to understand because imprinting has been used loosely to refer to a number of different mechanisms including psychological development, endocrinological actions of cells and protein-protein interactions. Genomic imprinting produces parent-of-origin effects. Parent-of-origin effects is a term that encompasses many of the non-traditional types of inheritance and other genetic and nongenetic mechanisms which show an effect depending on whether they were paternally or maternally derived.

\section{Genomic imprinting}

Genomic imprinting is one type of parent-of-origin effect that is recognized as differences in the phenotype depending on the transmitting parent. The first clues that genomic imprinting occurs in mammals came from studies in mice demonstrating uniparental disomy (UPD), transgene expression and pronuclear transplantation. It appears that genomic imprinting is not a permanent change in the DNA, but rather a temporary change related to expression that occurs due to specific modifications which in turn produce differential expression of the paternally and the maternally 
inherited alleles. The mechanisms by which genomic imprinting takes place are not fully understood but differential expression is associated with differences in methylation.

\section{UPD}

Parent of origin effects are particularly striking in some cases of UPD. UPD occurs when the two chromosomes (or parts of the two chromosomes) in a given pair come from only one parent. UPD has being recognized to occur with surprisingly high frequency. There are two types of UPD: isodisomy in which the two chromosomes in a pair come from only one parent and are genetically identical and uniparental heterodisomy in which the two chromosomes in a pair come from only one parent but are the different members of that pair. The distinction between uniparental isodisomy and uniparental heterodisomy is important, because it has implications for the time at which UPD arose and on the possibility of expressing autosomal recessive disorders. Two types of effects can be seen in UPD: (1) imprinting effects due to the lack of contribution from one parent - these result in changes of growth, behavior and intrauterine survival, as well as placental size and (2) autosomal recessive diseases in which one parent is a carrier for a recessive disorder and the offspring receives two copies of the abnormal gene. Studies of UPD are important because they may give information regarding salvage mechanisms for the correction of trisomies, increased risk for recessive disorders and areas of chromosomes with parentspecific expression of genes.

\section{Allelic expansion}

Parent-of-origin effects can also be seen in mutation rates, recombination rates and in a newly recognized type of mutation: allelic expansion. Allelic expansion is a mechanism by which a set number of DNA sequences is increased in each subsequent generation resulting in a specific phenotype. A number of different disorders such as Huntington disease, myotonic dystrophy, fragile $\mathrm{X}$ syndrome and others have been associated with allelic expansion. Parent-of-origin effects due to allelic expansion are evident in fragile $\mathrm{X}$ syndrome in which the expansion of the DNA sequences occurs only when the gene is transmitted through the mother. In Huntington disease, allelic expansion is greater when transmitted through the father and often results in an earlier and more severe onset of the disease.

\section{Mitochondrial inheritance}

Parent-of-origin effects can also be seen in disorders associated with mitochondrial inheritance. Mitochondrial DNA is maternally inherited, since it is inherited entirely through the egg cytoplasm. Thus mitochondrial diseases are exclusively inherited from the mother. It seems likely that other cytoplasmic elements will also show parent-of-origin effects. Centrosomes, for example, are essential for cell division and are thought to be exclusively paternally derived. 


\section{Loss of heterozygosity}

Parent of origin effects are associated with loss of heterozygosity in some cancers such as Wilms tumor, in which there is loss of maternal chromosome 11 , sarcomas in which there is loss of maternal chromosome 13, the Philadelphia chromosome translocation involving only paternal chromosome 9 and only maternal chromosome 22 and neuroblastoma with amplification of the maternal $\mathrm{N}-m y c$ gene.

\section{Environmental parent-of-origin effects}

Parent-of-origin effects can also refer to the maternal environment of the developing fetus. In this case it is important to mention the possibility of parent-of-origin transgenerational effects that may become apparent in future generations. The possible environmental influences affecting meiosis and subsequent gene expression have far-reaching implications.

\section{CONCLUSION}

In conclusion, parent-of-origin effects can be seen in genomic imprinting, human triploids, chromosomal deletions, UPD, mitochondrial inheritance and allelic expansion. Parent-of-origin differences are also seen in recombination, mutation rates, condensation (compaction), pairing, late replication and methylation patterns. Germ line modifications which are probably responsible for many parent of origin effects (gamete to gamete) may also demonstrate transgenerational effects. Parent-of-origin effects play an important role in the placenta, embryo, fetal growth and behavior and must be remembered when trying to make sense of an unusual phenotype or a rare association.

This is a timely symposium which will bring attention to new developments in areas of non traditional inheritance and the expression of human disorders.

\section{REFERENCES}

1. Barlow D: Imprinting: A gametes point of view. Trends Genet 1994; 10: 194-199.

2. Hall JG: Genomic imprinting and it's clinical implications. New England J Med 1992; 328: 827-829.

3. Nicholls RD: New insights reveal complex mechanisms involved in genomic imprinting. Am $\mathrm{J}$ Med Genet 1994; 54: 733-740.

Correspondence: Judith G. Hall, M.D., Department of Pediatrics, University of British Columbia and Children's Hospital, 4480 Oak Street Room 2D15, Vancouver, V6H 4 V4 Canada. 